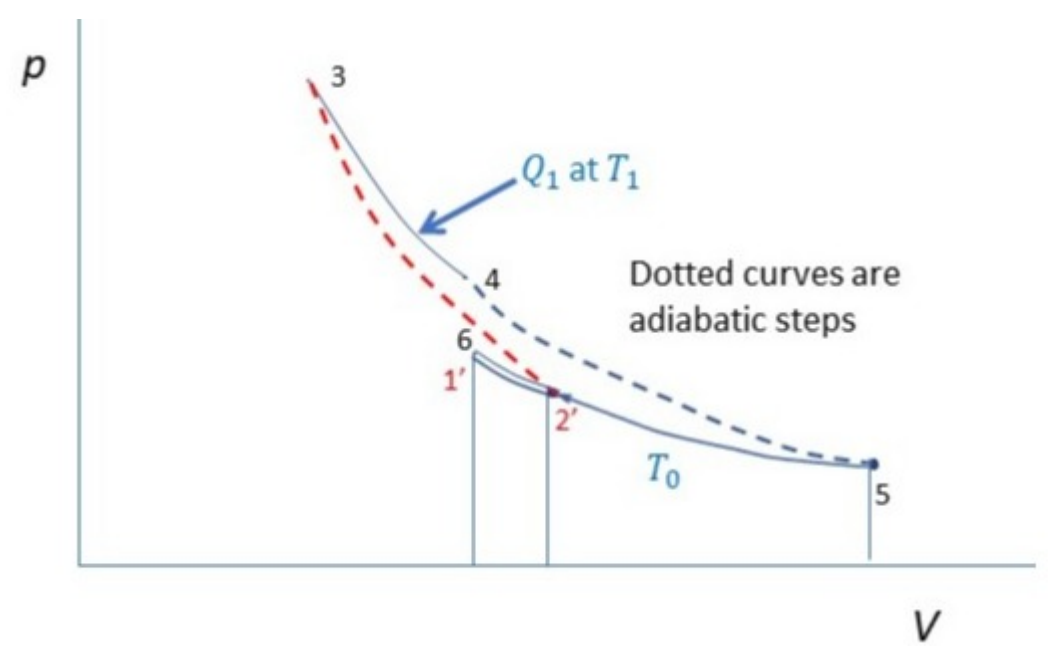

\title{
The Thomson-Clausius synthesis revisited: Why "conversion" of heat to work is a misnomer?
}

\author{
lin-shu wang ${ }^{1}$ \\ 1 State University of New York at Stony Brook
}

Funding: The author(s) received no specific funding for this work.

Potential competing interests: The author(s) declared that no potential competing interests exist.

\section{Abstract}

James Lovelock (2019) called the Newcomen-Watt-Carnot discovery of the motive power of heat the second "decisive event in the history of our planet," the event that led to the Thomson-Clausius synthesis of Carnot's and Joule's theories of heat introducing the conceptual tool of energy. "Whenever a new tool emerged within an endeavor, practitioners tended to use it in the context of previous habits and remained blind for a while to its full potential," observed Marshall McLuhan (1964). This paper argues that thermodynamic practitioners have been applying thermodynamics in the context of the pre-industrialization mechanical-sciences-correspondingly, treating connection and conversion of heat and work synonymously. The paper clarifies the relation between connection of heat and work and conversion of work to heat; two innovative highlights of the paper are the necessity of considering interventionist causation in the treatment of "processes of unnatural direction," and a new interpretation of the Clausius Cycle as "transmission transformation" drives/compensates the "extraction of heat" instead of the "conversion of heat," which implies that heat can be converted to work without "compensation." Furthermore, the paper makes the case for supplanting the conceptual tool of "energy" with the conceptual tool of "nature's statistical tendency" foretelling that the new conceptual tool will fulfill the full potential of the $18^{\text {th }}-19^{\text {th }}$-century discovery of the motive power of heat. 
Keywords: equivalence of heat and work, the Thomson-Clausius synthesis, energy, the Clausius Cycle, nature's statistical tendency.

\section{Introduction}

For two millenniums, human supplemented its human/animal-muscle-powers with power from natural mechanical processes in the forms of water wheels and windmills. Since early Homo sapiens, human had been relying on fire for heat and light for 300,000 years, and the practice continued in the last two millenniums. During these years, "heat" and "mechanical effects" were considered to be things of different categories, i.e., they were ontologically different. Most phenomena and processes were accounted for either in terms of supernatural forces or by various theories of motion. Then, scientific revolution took place; theories of motion were unified into Newton's laws of motion; force was the primary concept of Newtonian science while the word "energy" was not yet used as a scientific term; science and philosophy were based on the mechanical worldview.

Following that, a landmark event happened. As Lovelock put it, "...there have been two previous decisive events in the history of our planet. The first was about 3.4 billion years ago when photosynthetic bacteria first appeared. Photosynthesis is the conversion of sunlight to usable energy. The second was in 1712 when Newcomen created an efficient [steam engine] machine that converted the sunlight locked in coal directly into work" [i]. I'll also refer it as the Newcomen-WattCarnot (NWC) discovery of the motive power of heat. It is decisive, in addition to what Lovelock alluded to as marking the beginning of the Anthropocene, for the event being associated with several philosophical and scientific developments:

1. heat and mechanical energy are after all ontologically of the same category, an understanding derived from the MayerJoule equivalence principle;

2. thus, energy became a word in scientific lexicon, the significance of which is that heat energy, heat released from fuel combustion in addition to mechanical energy, is a new source for power;

3. synthesis achieved by Clausius and Thomson in 1850-54 of Carnot's theory of heat and the Mayer-Joule principle giving birth to thermodynamics, a new branch of physics, the energy physics of steam engines;

4. following thermodynamics, the development of statistical mechanics by Clausius, Maxwell, Boltzmann, Gibbs, and Poincare — which, together with quantum mechanics later, completed the transformation of the mechanical worldview to the statistical worldview.

The Thomson-Clausius synthesis gave rise to mechanistic thermodynamics. This paper focuses on the Thomson-Clausius synthesis and the cornerstone of the synthesis, the equivalence of heat and work. The paper attempts to explain why there have been critiques of mechanistic thermodynamics ${ }^{[i i]}$, specifically on equivalence principle ${ }^{[i i i]}$. Equivalence principle asserts symmetry between work to heat process and heat to work process as energy conversion processes, allowing only while mechanical energy can be converted entirely to heat, heat cannot be converted entirely to mechanical energy, i.e., work. Generations of students of thermodynamics have been indoctrinated by this energy conversion doctrine. It is a truism that is transformed into a "truth," while Job and Lankau characterized it this way, "Doubts about the meaning of the heat-work equivalence-accepted without question for one and a half centuries—appear hopeless and 
almost heretical" [3]:171-172). Despite the acknowledgement of hopelessness of their effort, they called the equivalence

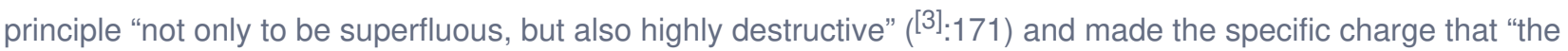
equivalence of heat and work assumed by the first law" assigns heat "a schizophrenic double role that it cannot fulfill" $([3]: 179-180)$.

The paper suggests that the genius of Thomson and Clausius lies in their realization that the historic advance of introducing the energy concept was intrinsically coupled with Carnot's insight of "a new force of nature found in nature's statistical tendency" so that an energy physics of steam engines was to be emerging from synthesizing Joule's theory (Joule's theory of heat is used synonymously here with the Mayer-Joule equivalence principle) with Carnot's theory. This resulted in the formulation of the two laws of thermodynamics, the first law and the second law. But their synthesis stopped short of according equal status to both theories (thus both laws). The synthesis contained a predisposition, not giving Carnot's theory its full due, by making Joule's theory to be synthesis' cornerstone. In doing so, it assigned heat "a double role that it cannot fulfill." This critique against the synthesis has also been made in A Treatise of Heat and Energy [iv]. That is, by Thomson's naming the theoryenergy physics the synthesis failed to understand the historic discovery of fuel-energy to be the discovery of, not a new form of "mechanical energy," but a new force of nature in the form of nature's statistical tendency found in fuel-energy. What we need today is a new synthesis of Carnot's theory and Joule's theory with the hindsight of statistical mechanics, i.e., we need to formulate a synthesis in accordance with a post-mechanical, statistical worldview—in which,

- symmetry of work-to-heat and heat-to-work is rejected, i.e., "though heat and work are ontologically the same category, physically [phenomenologically] they are of very different category" (in his Eighth Memoir, ${ }^{[v]}$ Clausius wrote, “... difference must exist between the conversion of work into heat, and the transformation of heat into work ...");

- connection and conversion are not always interchangeable: the required demarcation of the two, i.e., connection does not necessarily imply conversion, entails the abandonment of causal monism—a philosophical stricture that predisposed Thomson and Clausius in their synthesis in favor of Joule's theory (Sect. 2);

- Reconsideration of Clausius' "transformation theory" of heat is made to demonstrate that work is derived fromheat extraction rather than conversion of heat (Sect. 3);

- Demarcation of connection and conversion leads to the demarcation of the first and second laws, with the first law as the universal law of connection and the second law as the universal law of interventionist causation-i.e., a new synthesis of Carnot's and Joule's theories (Sect. 4);

In the new synthesis, the equivalence principle, with a correct take on the real meaning of the principle, is certainly not "superfluous" but finds its real role as partner of equal status with nature's statistical tendency for "making reversibility happen" (Sect. 5).

\section{Equivalence principle: connection or conversion of heat and work?}

\subsection{Causation, and preamble of the problem of connection vs. conversion}

Heat and work are obviously different phenomena. That was why they were thought to be categorially different. That is 
why the establishment of the Mayer-Joule principle was a historic advancement in physics when they were determined to be in fact of the same category ontologically. But their being of the same nature behind different "appearances" does not negate the significance of their being different phenomenologically. In many ways, the interpretation of the equivalence of heat and work as symmetry between heat and work is the surprising result of conflation or confusion of connection of heat and work with conversion of heat and work.

How to tell apart correlation vs. causation is a basic problem of "how to think scientifically," a lesson well known to every student. The phrase "correlation does not imply causation" refers to the logical fallacy to deduce a cause-and-effect relationship between two events or variables solely based on an observed statistical correlation between them. Connection vs. conversion is a lesser-known problem belong to how to tell different kinds of causations apart. Both connection and conversion have to do with causal relations: the problem here is why they are sometime used synonymously and other time we need to tell them apart. Hume famously addressed causation in science as efficient causation and causation in everyday life as causation with efficacy. Because mechanical sciences involve only causation of efficient causation, it is traditionally thought that Hume denied the existence of causation with efficacy. But that is very much an open question. It is certainly true that Hume concluded that giving efficacy to efficient causation is an anthropomorphic error: "He could not find in the natural occurrences of the material world the power and efficacy that he referred to in human affairs, and, as one of the greatest champions against anthropomorphism, warned against the projection of mind to objects" (vii]:34).

Sarasvathy in "Causation and effectuation"[vii], made an analysis of efficient causation and causation with efficacy and referred to them as causation and effectuation. Which she defined as:

Causation processes take a particular effect as given and focus on selecting between means to create that effect. Effectuation processes take a set of means as given and focus on selecting between possible effects that can be created with that set of means.

Sarasvathy wrote, "In economics and management theories, scholars have traditionally assumed the existence of artifacts such as firms/organizations and markets. I argue that an explanation for the creation of such artifacts requires the notion of effectuation. Causation rests on a logic of prediction, effectuation on the logic of control" $\left.{ }^{7}\right]$.

That is, natural phenomena or processes, existence of which is given, are explained in terms of the notion of efficient causation in accordance with Sarasvathy's definition of causation. But additionally, scientific theories can be applied for predicting new phenomena-successful prediction of new phenomena justifies the important role of hypotheses/theories in scientific method. Both extant phenomena and predicted new ones are inevitable or deterministic. Whereas artifacts such as firms/organizations and markets are not natural phenomena or processes. Unlike natural phenomena, the existence of firms/organizations and markets is not "given"; their existence is contingent. Economics theories and management theories, by identifying "a set of means," investigate how these artifacts came into existence historically by the application of the set of means - so that entrepreneurs can learn lessons of, with available means under their control, the creation of new artifacts. 
Thermodynamics, a branch of physics, is unique among physics. Carnot, the founder of thermodynamics, realized that unlike mechanical sciences of other physics branches, thermodynamics deals with artifact-machine of heat engines as well as natural phenomena, and how heat engines interacting with natural phenomena: in particular, how heat engines are powered by the natural phenomenon of heat flowing from high temperature to low temperature. Therefore, while causation for other branches of physics is under the stricture of efficient causation, Carnot realized, implicitly, that both efficient causation and effectuation, i.e., efficacious causation, are necessary in science and engineering. Carnot initiated a revolution against the Newtonian mechanical worldview with his theory. Ulanowicz, the American theoretical ecologist, described the philosophical significance of Carnot's theory with these words,

Most accounts of the history of science connect the demise of the Newtonian worldview to the advent of relativity and quantum theories near the turn of the $20^{\text {th }}$ century. But thermodynamicists are more inclined to reckon the departure as beginning some eight decades earlier, when Sadi Carnot described behaviors that did not belong in the conservative theatre that reversible Newtonian mechanics had erected. Contrary to Newton, every real change brings with it an inevitable loss. Carnot's experiments with simple physical systems later gave rise to mathematical constructs by Boltzmann and Gibbs that depicted a universe where loss was ubiquitous and ineluctable...

Everything ultimately degenerates into heat... [viii]

The allowance of efficacious causation is an implicit part of the philosophical implications of Carnot's theory, which of course includes the tenet that the world is irreversible. It is this central tenet that Carnot moved against the reversible worldview of Newton, thus in the irreversible world one finds nature's statistical tendency-the harness of which requires the concept of efficacious causation, explicitly or at least implicitly. Carnot foreshadowed the full-fledged statistical worldview of Boltzmann-Gibbs.

A note on terminology. Here we are talking about the metaphysics/epistemology of causation in science and engineering in Carnot's treatment while Sarasvathy is talking about causation in the context of clearly demarcated scientific theories and economics-management theories. As a matter of notation, it is preferrable to use in science and engineering efficient causation vs. efficacious causation $[6,4$, ix] —although the meanings of effectuation and efficacious causation are synonymous. In recent time, Zwier also finds in the structural foundation of thermodynamic theory efficacious causation, which she refers to as interventionist causation ${ }^{[x]}$. Here, efficacious causation and interventionist causation are used interchangeably in the context of science and engineering. (Zwier also refers efficient causation as "consensus view of physical causation [CVPC]. Here, efficient causation and CVPC are used interchangeably.)

The problem of connection (connexion) vs. conversion is intrinsically related to difference between efficient causation and efficacious/interventionist causation. While conversion is identified with processes of efficient causation, connection exists in conversion processes as well processes other than conversion processes-i.e., processes of plural causations. Unfortunately, the $19^{\text {th }}$ century of industrialization was also the century of foundationalism in which the mechanical philosophy and mechanical sciences, which allow only efficient causation, were at the height of their acceptance. Under this mechanical framework, the NWC discovery of the motive power of heat was treated by Thomson in terms of the 
"energy physics of steam engines" and connection and conversion were treated synonymously.

Citing Marshall McLuhan (1964), Ulanowicz noted,

Whenever a new tool emerged within an endeavor, practitioners tended to use it in the context of previous habits and remained blind for a while to its full potential. His [McLuhan's] example was IBM, which saw its purpose as the manufacture of business machines. It wasn't until its leaders realized they were in the business of processing information that the enterprise began to take off ${ }^{[x i]}$.

One can find a clue for the reason why energy physics treated connection and conversion synonymously in the "previous habits" of thermodynamicists.

\subsection{Discerning the real business of the Newcomen-Watt-Carnot discovery}

This paper argues that the Newcomen-Watt-Carnot discovery was another case-example of Marshall McLuhan's insight. The new tool was the motive power of heat, and the new conceptual tool was energy physics. The endeavor was, and still is, meeting human needs with the purpose of solving scarcity with the tool of energy physics. In this case, the previous habits/paradigm was mechanical sciences (the mechanical framework): the $19^{\text {th }}-20^{\text {th }}$ century causal monism, the technology of water wheels and windmills, and the science of mechanical energies and their inter-conversions. While the required paradigm is statistical sciences and causal pluralism [xii]. And the real conceptual tool is nature's statistical tendency (e.g., heat transfer from high temperature to low temperature; combustion of fuel and air resulting in heat release; radiative interaction of Sun, Earth, and space; etc.) - and its full potential and purpose, rather than supporting economic growth per se with the tool of energy physics, are creating sustainable economic growth and entropic orders with the tool of entropy physics. The paper aims for discerning the real business of the Newcomen-Watt-Carnot 17121824 discovery of the motive power of heat.

Let's begin with reviewing what was the previous habits/paradigm of thermodynamic practitioners. Since the 1 th century scientific revolution, an understanding of a reversible world that can be idealized as a conservative mechanical macrosystem made of conservative mechanical systems/devices had become well established. During this period, ".... the term 'energy' did not have a precise scientific definition, and most physicists thought matter [mass] and force were the only concepts needed to understand the natural world" ([xiii]:1). Human derived power from water wheels and windmills as well as animal muscle power. The working of these machines was based on the science of mechanics in terms of mass, Cartesian coordinates, and force. But from the very beginning of mechanics there has been a parallel track to the forcecentral framework, the energy-central framework. It was Leibniz during 1676-1689 who first attempted a mathematical formulation of the kind of energy which is connected with motion (kinetic energy). Leibniz noticed that in many mechanical systems (of several masses, $m_{i}$ each with velocity $v_{i}$ ),

$$
\sum \gtrless m_{i} v_{i}^{2}
$$

, was conserved so long as the masses did not interact. He called this quantity thevis viva or living force of the system. The recalibration of vis viva to 


\section{$1 / 2 \sum \not m_{i} v_{i}^{2}$}

, which can be understood as finding the exact value for the kinetic energy to work conversion constant, was largely the result of the work of Gaspard-Gustave Coriolis and Jean-Victor Poncelet over the period 1819-1839. The former called the quantity quantité de travail (quantity of work) and the latter, travail mécanique (mechanical work).

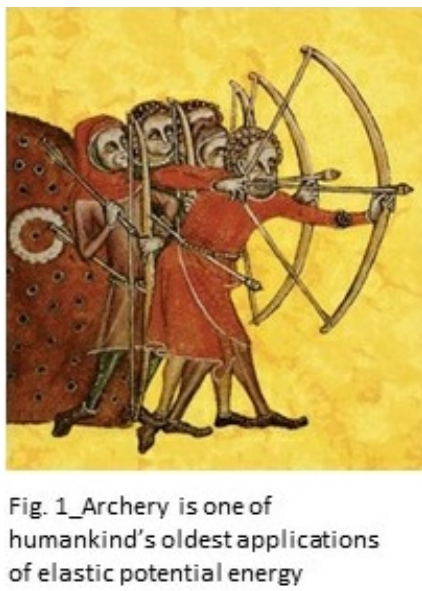

Although the term of "potential energy" was attributed to Rankine in the 19 century later, the scientific concept of potential energy can be found (in the tradition of "the energy-central framework") in the earlier work of d'Alembert's principle and in the formulation of Lagrangian mechanics in terms of mass, generalized coordinates and energy. The technological use of potential energy was even much older since the ancient time (see Fig. 1). Let's call the period before the advent of steam engines the pre-industrialization time, the time human derived power from water wheels and windmills as well as animal muscle power. We may make use of the concepts of kinetic energy and potential energy, i.e., mechanical energies, to review how a water wheel was understood in terms of mechanical energies.

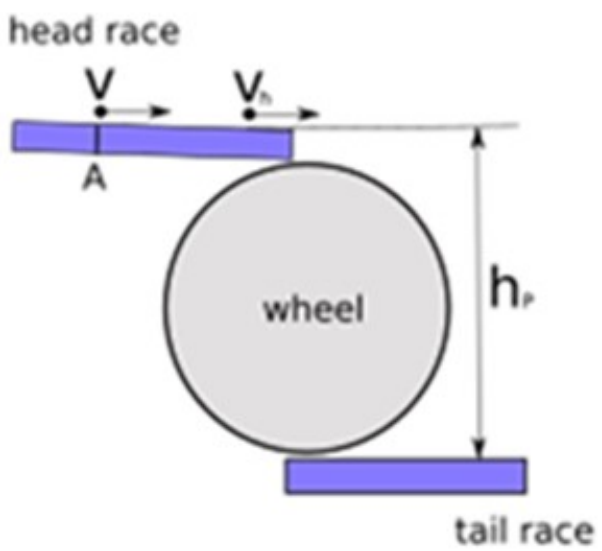

\section{Figure 2_Parameters for measuring the head and flow rate of a water wheel}

A water wheel is a mechanical system. In ideal mechanical systems, which are referred to as conservative systems or systems of conservative forces in physics, difference of kinetic energy and potential energy between head race and tail race (see Fig. 2) of a water wheel equals the water wheel work output. Power, the rate of work output, of the water wheel 
is,

$P=\Delta W / \Delta t=\rho \cdot g \cdot\left[\zeta_{h}+\left(h_{h}-h_{t}\right)\right] \cdot(\Delta q / \Delta t)$

Where

$A=$ cross sectional area of the channel $\left(\mathrm{m}^{2}\right), A_{h}$ head cross sectional area and $A_{t}$ tail cross sectional area

$g$ = strength of gravity $\left(9.81 \mathrm{~m} / \mathrm{s}^{2}=9.81 \mathrm{~N} / \mathrm{kg}\right.$

$h=$ pressure head $(m), h_{h}$ pressure head at head and $h_{t}$ at tail

$\dot{m}=\rho \cdot \dot{q}$

$P=\Delta W / \Delta t=$ power $($ Watt $)$

$v=$ velocity

$\dot{q}=\Delta q / \Delta t=$ volume flow rate $\left(=A \cdot v, \mathrm{~m}^{3} / \mathrm{s}\right)$

$W=$ work (joule)

$z=$ velocity head $\left(=\frac{v^{2}}{2 g^{\prime}}, m\right)$

$\eta=$ efficiency $=1.0$ for conservative systems

$\rho=$ density of water $\left(1000 \mathrm{~kg} / \mathrm{m}^{3}\right)$

That is, mechanical energy — the kinetic energy represented by difference in velocity head $z_{h}$ - 0 , and the potential energy represented by difference in pressure head, $h_{h}-h_{t}$-gives rise to work in an idealized water wheel.

Both the ideal Newtonian mechanics and the Lagrangian and Hamiltonian mechanics deal with conservative mechanical systems. A real mechanical system such as a real water wheel can be readily addressed with Newtonian mechanics by the assumption of friction forces, which accounts for the departure of the performance of a real system from the idealized conservative system. Such consideration can be summarized in terms of efficiency, $\eta$. That is, for a real water wheel, $P=\eta \cdot \rho \cdot g \cdot\left[\zeta_{h}+\left(h_{h}-h_{t}\right)\right] \cdot(\Delta q / \Delta t)$

The pre-industrialization understanding of how work and power were derived is this:

1. The world can be idealized as a conservative, reversible world

2. Departure from the idealization in the real world can be accounted for by frictional force and effects

3. Energy's convertibility: In this idealized world, mechanical energy of a given form can be entirely converted into another form, and into work, and vice versa

4. Energy's capacity for work: In view of Eqns. (1) and (2), mechanical energy is the capacity of a system for doing work.

5. Causal monism: Physics knowledge rested on the metaphysics and epistemology of efficient causation and physical necessity, or what we may call causal monism, or what Zwier calls "consensus view of physical causation" (CVPC) [10]. 
These five points define the previous paradigm of science and engineering when Newcomen-Watt-Carnot made their discovery that heat can be a source for the production of power. Thanks to Carnot, the first two points have been completely revised since the NWC discovery: our world is fundamentally irreversible, and besides frictional phenomena we have examples of heat transfer and diffusion/mixing which additionally account for irreversibility-departure of the real world from a reversible conservative world. However, the rest three points remain the previous habits/paradigm that conditioned the mind of thermodynamicists, which continues to this day.

Jump out at our eyes is the standard definition of energy:energy is the capacity of a system for doing work. Before the NWC discovery, the science of mechanics did not need an independent definition of energy. While mechanical energy of a system was indeed the capacity of the system for doing work, the mechanical-energy framework was an alternative to the force framework, an option for the science of motion. The discovery of the motive power of heat made it anecessity to introduce the concept of energy that comprises of heat energy and mechanical energies for the science of motion and heat. The resulting energy-centric, energy physics is completely different from mechanics. Both the meaning and the role of energy are now different.

Whereas kinetic energy and potential energy are ontologically and phenomenologically of the same category (in the sense that they can be converted entirely into each other under idealization), heat (heat energy) and work (mechanical energy) are not phenomenologically of the same category. The necessary introduction of the term, energy, changed the meaning of the term and what energy was capable of. Little of the humongous amount of energy in an ocean is capable of doing work. Therefore, the standard definition of energy, energy is the capacity of a system for doing work(holdover from Point 4), and symmetry between heat and work (holdover from Point 3), are both non-sensical.

\subsection{Methodological pluralism, interventionist theory of causation}

The NWC discovery as well as the Mayer-Joule-Thomson-Clausius introduction of the energy concept were clearly revolutionary. It is surprising that these holdovers survived the revolutionary "creative destruction." One possible explanation was that these historic developments took place in the $19^{\text {th }}$ century, which was at the peak of foundationalist thinking (holdover from Point 5, which may explain the surprising holdovers 3 and 4). In fact, foundationalism continued well into the $20^{\text {th }}$ century in the form of logical positivism—which declined only after the postmodernist movement. In comparison to the postmodernist movement, Carnot's challenge to Newtonianism and the Thomson-Clausius synthesis' challenge to force-centric mechanistic physics, insightful scientifically that they are, lack the social force of the postmodernist movement. It took the new historicists, Norwood Russell Hanson, Thomas Kuhn, and Paul Feyerabend, in what Nickles calls the "Battle of the Big Systems" of the 1960s to deliver devastating knockout against logical empiricism [xiv]. By 1967, John Passmore reported, "Logical positivism, then, is dead, or as dead as a philosophical movement ever becomes" ([xv]:57).

Before that point, thermodynamic thinking had been under the sway of methodological monism and causal monism of 
scientific method, which perfectly described the idealized world of mechanical energies. But which made discerning the real business of the NWC discovered world all but impossible. In the old, idealized world, conversion among mechanical energies is governed, or inevitably connected, by equations of motions of efficient causation, such as equations (1) and (2). The same also applies to conversion of mechanical energy into heat. These processes are deterministic and inevitable, i.e., their final states are given. Connection and conversion are synonymous. However, processes of heat to work belong to a different kind of processes (see Sect. 3.3). They cannot be treated by an equation of motion. There is no equation of motion. Nor is the final state inevitable. The system before the event and that after the event are connected, but not inevitably connected, by equivalence principle. The system before the event cannot be said to the cause of the system after the event. What drives the event is "nature's tendency in the system" before the event. But, as it'll be discussed in Sect. 5, we still cannot say that nature's tendency in the system to be the sole cause of the event. Before that discussion, it is necessary to first recognize that the treatment of heat to work processes entails for us to allow causation other than CVPC.

James Woodward made an important contribution to the philosophy of causation with the development of his interventionist theory of causation ${ }^{[x v i]}$. Zwier made the case that "interventionist reasoning constitutes the structural foundation of thermodynamic theory, and that thermodynamic theory can provide clear answers to meaningful questions about whether or not a certain variable is a cause of another in a given context" [10]. Zwier described the interventionist structures especially in Clausius' treatment of the second law of thermodynamics in her Ph.D. thesis this way,

In the background of his 1854 paper is the awareness that the science of heat is entirely about "transformations". These transformations can be divided into two types: (1) simple transfers of heat from one body to another, and (2) conversions from work to heat or vice versa. Each of these two types of transformations can be carried out in what we might call either a natural or an unnatural direction. For example, we see many instances around us of heat transfer from a warm body to a cooler one; this is the spontaneous direction of heat transfer. Likewise, in regard to the second type of transformation, we see many instances around us of work generating heat-for example, the friction of movement spontaneously results in heat. However, it is possible to artificially force the unnatural version of each process to occur. This is exactly what a heat engine does, for example: it "converts" heat into work. Analogously, we can artificially force an unnatural heat transfer, making heat travel from a cold body to a warmer one. In these latter two artificially-induced scenarios, however, we use a "compensating" process to drive the unnatural one. In a heat engine, the unnatural conversion from heat to work occurs only when accompanied by the natural direction of heat transfer from hot to cold. Likewise, a heat engine running in reverse accomplishes the unnatural direction of heat transfer (from cold to hot), but this is accompanied by the natural conversion from work to heat. $\left[{ }^{10]}: 133-134\right)$

More details of Clausius' treatment can be found in the source of Zwier's thesis, the elucidating paper by William Cropper, "Rudolf Clausius and the Road to Entropy" [xvii].

Clausius clearly recognized that "conversion' of heat into work" is an unnatural "conversion transformation" that requires "compensating transmission transformation" of natural heat transfer from hot to cold to make it happen, an 
interventionalist concept, whereas the standard notion of 'conversion' is a CVPC concept. For the former, connection and conversion are not interchangeable while for the latter, they are. However, Clausius' interventionalist thinking was implicit, and he also agreed with Joule and Thomson that energy or heat can be converted into work:

Equivalence of Heat and Work: In all cases where work is produced by heat, a quantity of heat proportional to the work done is expended; and inversely, by the expenditure of a like quantity of work, the same amount of heat may be produced (Clausius ${ }^{\left[{ }^{2} \text { iii] }\right.}$ ).

By using the terms of "expended" and "expenditure," he did not separate the connection of heat and work from the conversion of heat into work explicitly.

On the other hand, in using the terms of "expended" and "converted," Clausius was explicit in rejecting symmetry in the equivalence of heat and work (see ${ }^{[5]}$ ), an important part of his thinking on equivalence principle that has been overlooked by other thermodynamicists, including Job and Lankau. ${ }^{[3]}$ His interventionist 'transformation theory' of heat in the development of the entropy concept is the "most important, and at the same time least understood, part of the Clausius story" ([17]:1068). Clausius' treatment was closer to Carnot's than the energetic position taken by Joule and Thomson, but his treatment was subtle and an analysis of which is given in Sect. 3.

\section{The Clausius cycle}

Coppersmith described the problem faced by Thomson and Clausius in 1850 as Thomson's Problem: "the conflict between Carnot and Joule. for work to be generated, Joule required the consumption of heat and Carnot required the transfer of heat—but Clausius saw that it wasn't a case of either/or—both requirements could be met" [xix].

\subsection{Clausius' resolution of Thomson's problem}

Clausius began with Carnot's invention of a four-stage reversible cycle of isothermal heat addition, adiabatic expansion, isothermal heat-rejection/compression, and adiabatic compression. ${ }^{[\mathrm{xx}]}$ In the modern thermodynamic understanding this is a reversible cycle with a constant entropy falling flow from a high temperature to a low temperature. But Carnot's original understanding was that of caloric theory that heat is a conserved entity, thus a constant heat falling from a high temperature to a low temperature. Clausius realized that Carnot's "transfer of heat" requirement can incorporate the Joule requirement that in the falling heat flow heat is not a constant, i.e., heat is converted.

Clausius proposed a six-stage reversible cycle. In the following I adopt the terms and notations of William Cropper in his 1986 article Rudolf Clausius and the road to entropy $\left[{ }^{17}\right]$, with two exceptions: following Zwier's revised adoption of the Cropper treatment the orders of $Q_{2}$ and $Q_{1}$, and $t_{2}$ and $t_{1}$ are inversed; the details of Clausius' reasoning that resulting in the resolution of the mathematical form of equivalence value are passed over so that I go directly to express the "condition of compensation" in terms of equivalence value $Q / T$. 


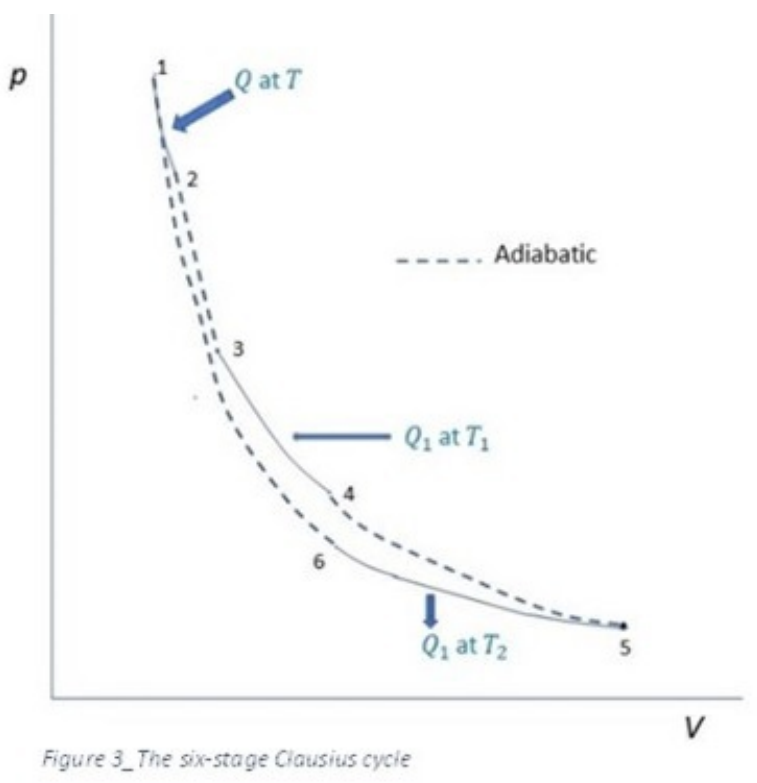

The Clausius cycle's six steps are then as follows (as shown in Fig. 3):

Step 1, an isothermal expansion at the temperature $T, 1 \rightarrow 2$, in which the heat $Q$ from a heat reservoir at $T+d T$ is supplied to the working fluid system.

Step 2, an adiabatic expansion, $2 \rightarrow 3$, in which the temperature decreases from $T$ to $T_{1}$.

Step 3, an isothermal expansion at $T_{1}, 3 \rightarrow 4$, in which the heat $Q_{1}$ from an energy source is supplied to the working fluid.

Step 4 , an adiabatic expansion, $4 \rightarrow 5$, in which the temperature decreases from $T_{1}$ to $T_{2}$.

Step 5, an isothermal compression at $T_{2}, 5 \rightarrow 6$, in which heat $Q_{1}$ is discharged by the working fluid to the reference-heat-sink reservoir at $T_{0}=T_{2}-d T$.

Step 6, an adiabatic compression, $6 \rightarrow 1$, in which the temperature increases from $T_{2}$ back to $T$.

Clausius designed the six-stage cycle with the heat $Q_{1}$ from a heat source system at $T_{1}$ falling from $T_{1}$ to $T_{2}$ discharged at Step 5. This falling of heat, which is called a natural "transmission transformation" by Cropper, "drives" the unnatural "conversion transformation" of $Q$ into $W$ work. Fig. 3 shows how the cycle affects the pressure and volume of the working-fluid system, and that $W(=Q)$ is represented by the $p-V$ area enclosed by 1-2-3-4-5-6-1.

Clausius proposed this six-stage cycle in his 1854 "fourth memoir" $\mathrm{f}^{0}$ ] formulating the "transformation theory" of heat, which initiated the process leading to his eventual 1865 publication of the paper announcing the concept of entropy and the entropy principle. Cropper noted, “... his place in the beautiful clear line of development of thermodynamics between 1824 and 1875-from Carnot to Clausius, and then to Clausius' greatest successor, Willard Gibbs. Clausius's role in this was pivotal" [ $\left.{ }^{17}: 1073\right]$. To the scientific story of this glorious achievement this paper has nothing to add, except that the resulting entropy principle has been universally viewed as a selection principle-an issue that will be considered in Sect. 4. 
Here in this section, it addresses the engineering story of Thomson's problem, how Clausius resolved the problem of incorporating both "transfer of heat" and "consumption/conversion of heat" by the six-stage cycle. Clausius' analysis led to the conclusion of the condition of compensation, the balance between the natural transmission transformation and unnatural conversion transformation, to be

$$
\frac{Q_{1}}{T_{1}}-\frac{Q_{1}}{T_{2}}+\frac{Q}{T}=0
$$

Specifically, what is the meaning of the "transfer of heat of the amount $Q_{1}$ " driving the "conversion of heat $Q$ into work of the same amount"? According to the condition of compensation (3), $Q$ (i.e., the amount of work $W$ ) equals $T\left(\left[1 / T_{2}\right]\right.$ $\left.\left[1 / T_{1}\right]\right) Q_{1}$. That is,

$$
Q=T\left(\frac{1}{T_{2}}-\frac{1}{T_{1}}\right) Q_{1}
$$

Let's break down the question into components.

What is exactly the meaning of conversion or consumption of heat energy (thermal energy)? The latter term of consumption is particularly problematic since energy is conserved; it can only mean that a form of energy is consumed. In that take, the former term of conversion is a better term. So, the question is what the meaning of conversion is.

This then leads to the issue: given that the drive in the Clausius cycle is given, $\left.\left(1 / T_{2}\right]-\left[1 / T_{1}\right]\right) Q_{1}$, why the converted heat in accordance with (4) is proportional to $T$, the temperature of the converted heat? Since heat at different temperatures corresponds to heat of different forms. The situation suggests the form of the converted heat plays a role in its conversion -a suggestion that $\left(\left[1 / T_{2}\right]-\left[1 / T_{1}\right]\right) Q_{1}$ is not the sole drive of the Clausius cycle. It follows that the compensation or the balance may exist as Clausius visioned between $\left(\left[1 / T_{2}\right]-\left[1 / T_{1}\right]\right) \cdot Q_{1}$ and $Q / T$, but the meaning of $\left(\left[1 / T_{2}\right]-\left[1 / T_{1}\right]\right) Q_{1}$ as the drive of conversion of heat, $Q$, into $W$ is ambiguous.

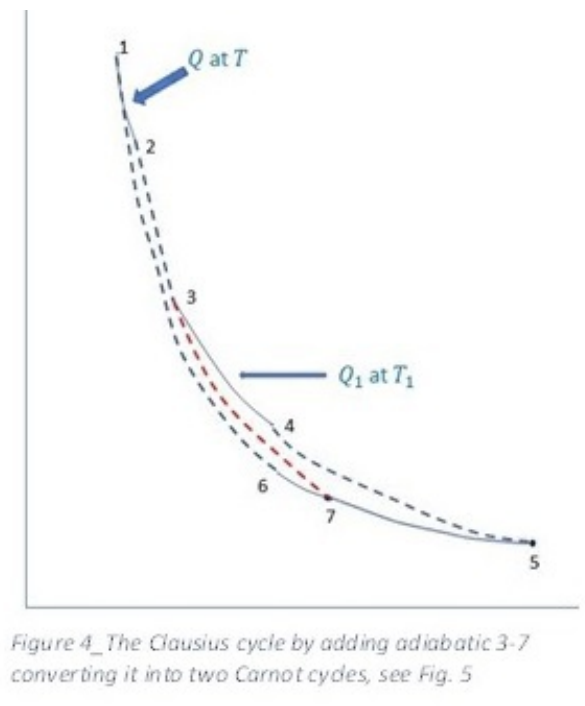


Looking at the problem as a conversion problem, the only valid interpretation is the original-Carnot-cycle interpretation of the Clausius cycle as being made of two Carnot cycles, as shown in Fig. 4. This is then plotted into two separate Carnot cycles, as shown in Fig. 5.

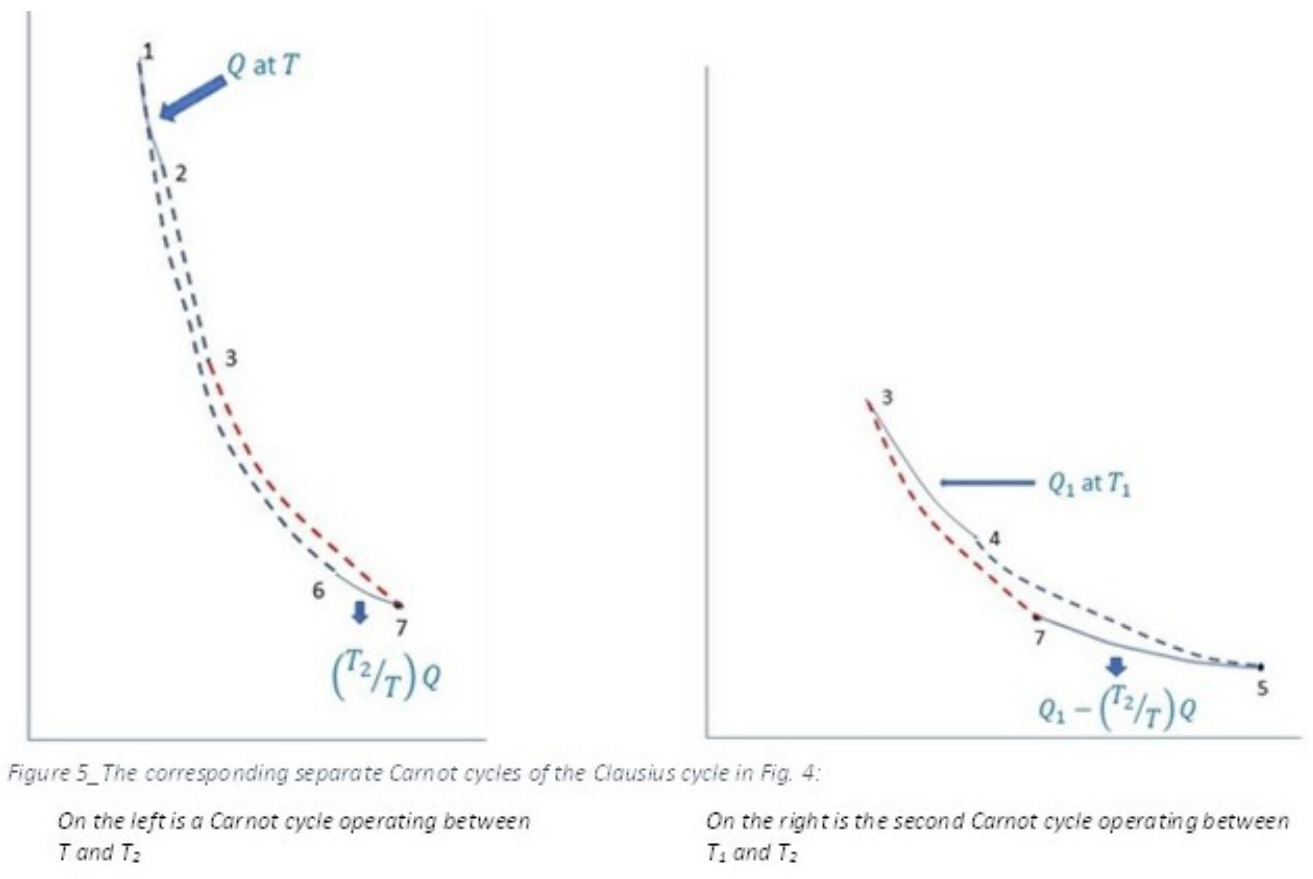

As shown on the left of Fig. 5, its working fluid discharges heat of the amount of $\left.T_{2} / T\right) Q$. Subtracting this amount from $Q_{1}$ of the original heat discharging of 5-7-6, we have the working fluid discharging, during Step 5-7 of the second Carnot cycle, $Q_{1}-\left(T_{2} / T\right) Q$. Given the condition of compensation of the Clausius cycle, $Q=T\left(\left[1 / T_{2}\right]-\left[1 / T_{1}\right]\right) Q_{1}$, the discharged heat during Step 5-7 is consistent with Carnot' theory applied to the second cycle:

$$
\frac{Q_{5-7}}{T_{2}}=\frac{Q_{1}-T_{2} / T Q}{T_{2}}=\frac{Q_{1}-T_{2}\left(\frac{Q_{1}}{T_{2}}-\frac{Q_{1}}{T_{1}}\right)}{T_{2}}=\frac{T_{2} \frac{Q_{1}}{T_{1}}}{T_{2}}=\frac{Q_{1}}{T_{1}}
$$

Figures 4 and 5 confirm that the "conversion interpretation" of the Clausius cycle is reducible to Carnot cycles. This would have been a disappointing conclusion. It is the same conclusion in accordance with the universally held "doctrine of energy conversion of Joule and Thomson," which leads to three logically falsifiable inferences:

1. Conversion of heat into work is always accompanied with a required heat discharging in accordance with the Carnot cycle, therefore, heat cannot be entirely converted to work. This last statement was rejected by Planck, [[i]:81-82] details for the reason of rejection are given in A Treatise of Heat and Energy $\left[{ }^{4}\right]$.

2. In accordance with the Carnot cycle, more heat can be converted into work with heat reservoir at lower temperature. Again, this is an inference based on the situation of a Carnot cycle instead of a universally true statement; an explanation is given in Sect. 3.2 setting straight the impact of a heat reservoir.

3. Entropy pessimism as based on the inference of the inevitability of entropy growth leading to the corollaries of "inevitable accumulation of heat" and "inevitable increase of global entropic disorder." In view of Falsifiable Inference $\# 1$, the conclusion of inevitable accumulation of heat is not true. Paper [9] also argues against the conclusion of 
inevitable increase of global entropic disorder.

That is, the extraordinary inventiveness of Carnot cycle by Sadi Carnot is an uncut diamond—its brilliances are hidden by imperfections that in the wrong hands can lead to misuse and misinterpretation. The genius of Clausius was by proposing the Clausius cycle he unfolded a great use of the Carnot cycles leading to "the road to entropy' $\left[{ }^{18}\right]$.

Here, I want to suggest a second use that manifests the genius of the Clausius cycle: Consider having theT-isotherm, 12, of Fig. 3 or Fig. 4 lowered to isotherms, 1'-2', at the temperature of the isotherm 5-6 as shown in Fig. 6a. This change makes the first Carnot cycle in Fig. 5 disappeared and, correspondingly, the Clausius cycle reduces to the second Carnot cycle as shown in Fig. 6a. We then lowering 1'-2' slightly to be at temperature of $T_{2}-d T$ suggesting 1'-2' to be an isothermal expansion process that extracts heat from a $T_{2}=T_{0}$ heat reservoir as shown inFig. $6 \mathbf{b}$.

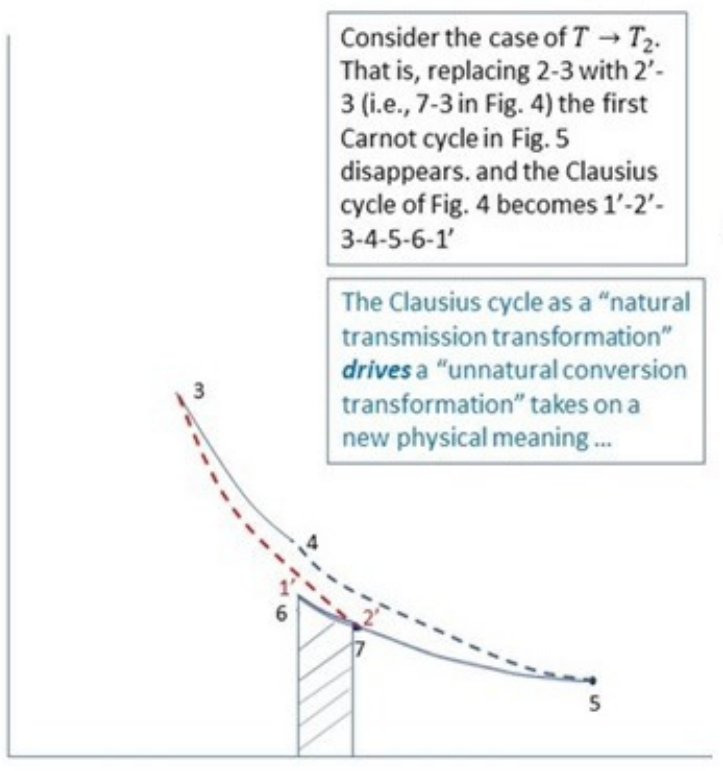

Figure 6a_The Clausius cycle with Step one, 1-2, becoming Step One, $1^{\prime}-2^{\prime}$, at temperature $T_{2}$

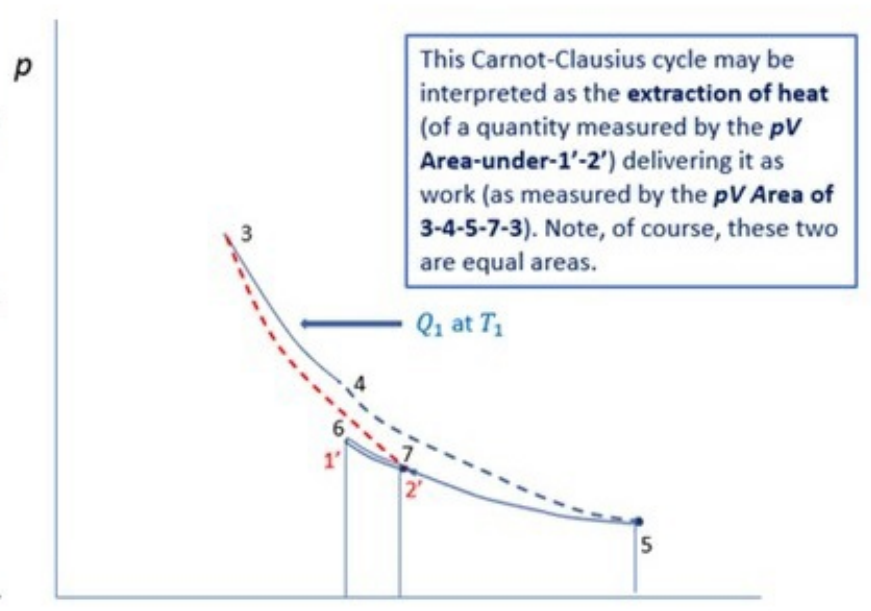

Figure 6b_The Carnot-Clausius cycle with $T_{0}$-reservoir $\left(T_{0}=T_{2}\right)$

Now the five steps of this Clausius cycle, which is called as a "five-stageCarnot-Clausius cycle" for differentiating it from the general cases of the Clausius cycle, are as follows: 
Step 1, an isothermal expansion at $T_{1}, 3 \rightarrow 4$, in which the heat $Q_{1}$ from an energy source is supplied to the working fluid.

Step 2 , an adiabatic expansion, $4 \rightarrow 5$, in which the temperature of the working fluid decreases from $T_{1}$ to $T_{2}+d T$.

Step 3, an isothermal compression at $T_{2}+d T, 5 \rightarrow 6$, in which heat $Q_{1}$, the same amount of Step 1, is discharged by the working fluid at temperature $T_{2}+d T$ to the reference-heat-sink reservoir at $T_{2}\left(=T_{0}\right)$.

Step 4 , following an infinitesimal $6 \rightarrow 1^{\prime}$ (which is not counted here as a step) in which the working fluid temperature drops from $T_{2}+d T$ to $T_{2}-d T$, an isothermal expansion at the temperature $T_{2}-d T\left(=T_{0}-d T\right), 1^{\prime} \rightarrow 2^{\prime}$, in which the heat $Q^{\prime}=T_{0}\left(\frac{1}{T_{2}}-\frac{1}{T_{1}}\right) Q_{1}$ is extracted from a heat reservoir at $T_{2}=T_{0}$ by the working fluid system.

Step 5 , an adiabatic compression, $2^{\prime}(7) \rightarrow 3$, in which the temperature increases from $T_{2}-d T$ back to $T_{1}$.

By giving up the "doctrine of energy conversion of Joule and Thomson," we find the true physical-meaning of the Clausius cycle:

given a transmission-transformation-drive of $\left(\left[1 / T_{2}\right]-\left[1 / T_{1}\right]\right) Q_{1}$, a reversible heat engine cycle as prescribed by the Carnot cycle or the Clausius cycle operates, not as instruction for the conversion of heat, but for the extraction of heat from a $T_{0}$-heat-reservoir of the amount of $T_{0} \cdot E G P \equiv T_{0} \cdot\left\{\left(\left[1 / T_{2}\right]-\left[1 / T_{1}\right]\right) Q_{1}\right\}$ delivering it as work of equal amount.

Extraction of heat may take place from the reference heat reservoir at $T_{0}$ as the present case of the Carnot-Clausius cycle - or, from an available heat reservoir at $T_{X}$ for a general Clausius cycle.

Such a general case of "Clausius cycle with- $T_{X}$-reservoir" is described by the six steps: 
Step 1 , an isothermal expansion at $T_{1}, 3 \rightarrow 4$, in which the heat $Q_{1}$ from an energy source is supplied to the working fluid.

Step 2 , an adiabatic expansion, $4 \rightarrow 5$, in which the temperature decreases from $T_{1}$ to $T_{2}$.

Step 3 , an isothermal compression at $T_{2}, 5 \rightarrow 6$, in which heat $Q_{1}$ is discharged by the working fluid to the reference-heat-sink reservoir at $T_{0}=T_{2}$.

Step 4 , an adiabatic compression, $6 \rightarrow 1_{x}$, in which the temperature increases from $T_{2}$ to $T_{x}-$ $d T$, where $T_{x}$ is the temperature of an available heat reservoir.

Step 5 , an isothermal expansion at the temperature $T_{x}-d T, 1_{x} \rightarrow 2_{x}$, in which the heat $Q_{x}=$ $T_{x}\left(\frac{1}{T_{2}}-\frac{1}{T_{1}}\right) Q_{1}$ is extracted from the $T_{x}$-heat-reservoir by the working fluid system.

Step 6 , an adiabatic compression/expansion, $2_{x} \rightarrow 3$, in which the temperature increases/ decreases from $T_{x}$ back to $T_{1}$.

The earlier issue concerning the nature of compensation or balance between $\left(\left[1 / T_{2}\right]-\left[1 / T_{1}\right]\right) \cdot Q_{1}$ and $Q$ is answered. Different amount of produced work is not the result of a "drive-contribution from $Q_{x}$ at $T_{x}$ " as suggested by Fig. 5. For all cases, the drives are the same drive of EGP $=\left(\left[1 / T_{2}\right]-\left[1 / T_{1}\right]\right) \cdot Q_{1}$. The same drive leads to specific amount of extracted heat, $T_{X} \cdot\left(\left[1 / T_{2}\right]-\left[1 / T_{1}\right]\right) \cdot Q_{1}$, as a function of the available heat reservoir's temperature, $T_{X}$; these differences in reservoir temperatures produce different amounts of produced work.

Every Carnot cycle should be understood in terms of the Carnot-Clausius cycle with $T_{0}$-reservoir as shown in Fig. 6b, in which the produced work is derived from extracted heat.

\subsection{The general concept of heat extraction}

$\left(\left[1 / T_{2}\right]-\left[1 / T_{1}\right]\right) Q_{1}$ is to be called entropy growth potential (EGP), see $A$ Treatise for a general definition of which. This understanding of "the produced work being derived from extracted heat"

$W=T_{0} \cdot E G P$

has been proposed earlier in $A$ Treatise, $\left[{ }^{4}\right]$ not in the context of the Clausius cycle but based on an insight of Poincare, who wrote,

[These thermodynamic laws] can have only one significance, which is that there is a property common to all possibilities; but in the deterministic hypothesis there is only a single possibility, and the laws no longer have any meaning. In the indeterministic hypothesis, on the other hand, they would have meaning, even if they were taken in an absolute sense; they would appear as a limitation imposed upon freedom ([xxii]:122-123).

That is, one can define a Poincare range with a two bookend-limits of the "single possibility of spontaneous event" (e.g., a natural heat transmission event) and the "corresponding reversible event" (a perfect unnatural-transformation-event). Any specific event within the range is a possible event. The total entropy growth of the spontaneous event limit, for the setting 
of a Carnot cycle between $T_{1}$ and $T_{2}$, is $-\left(Q_{1} / T_{1}\right)+\left(Q_{1} / T_{2}\right)$ (see $A$ Treatise [4] for details). It has been shown that this "total entropy growth" defines the entropy growth potential (EGP) for the Poincare range: EGP is the universal drive for every event in each Poincare range including the reversible event—for which the produced work equals, $W=T_{0}\left(=T_{2}\right) \cdot E G P=T_{0} \cdot\left(\left[1 / T_{2}\right]-\left[1 / T_{1}\right]\right) \cdot Q_{1}$.

Note that in (5) the value of EGP is dependent of $T_{0}$, i.e., EGP itself is a function of $T_{0}$, EGP $=f\left(T_{0}\right)$. The Falsifiable Inference \#2 is explained by the fact that for a Carnot-Clausius cycle with a $T_{0}$-heat-reservoir as both its heat sink and its heat reservoir, EGP increases with lower $T_{0}$, while the amount of extracted heat is proportional to $T_{0}$, thus, decreases with lower $T_{0}$. But while the extracted heat decreases linearly with lower $T_{0}$, EGP increases at a steeper rate with lower $T_{0}$, resulting in a net increase in the product of the two terms, $T_{0}$ and $f\left(T_{0}\right)$, for the produced work.

Equation (5) is applicable to all cases of thermodynamic work-source systems with statistical tendency towards equilibrium in interaction with a heat reservoir. A similar finding for all cases of isolated thermodynamic composite systems with statistical tendency towards internal equilibrium (independent of any external heat reservoir) has also been made ${ }^{[23,4]}$.

Such systems are overlooked by the theory of exergy in the engineering literature because exergy is defined as "the energy that is available to be used." Isolated systems, in their changes toward internal thermodynamic equilibrium, involve no change in energy. By "definition," therefore, isolated systems are not the kind of systems of interest to the theory of exergy. This is, of course, a totally erroneous energetic way of looking at the problems. Given an isolated composite system, whether it is an example of free expansion $\left[{ }^{23,4}\right]$ or a pure diffusion process $\left.{ }^{4}\right]$ or a heat transfer between internal components of a thermal composite system $\left[{ }^{23,4}\right]$, reversible work can be derived from its EGP, in accordance with the principle of increase of entropy, of the amount,

$W=T_{\text {Reservoir }} \cdot E G P$

In these cases, EGP is independent of the reservoir temperature $T_{\text {Reservoir; }}$ so, similar to the case of a "Clausius cycle with- $T_{\mathrm{X}}$-reservoir," the reversible work can be derived from any available heat reservoir of arbitrary temperature, $W=T_{X \text { - }}$ Reservoir - EGP.

In every isolated system, its EGP enables reversible extraction of heat, of amount equaling the product of EGP andT $T_{X}$ Reservoir (of an available reservoir), converting it entirely (100\%) to mechanical work. This falsifies Falsifiable Inference \#1, which is still widely held to be a corollary of the second law or a statement of the second law (see ${ }^{[x i v]}$ ).

\subsection{Three kinds of processes that are grouped into two groups}

To recapitulate. There are three kinds of processes:mechanical processes, processes of natural direction, and processes of unnatural direction. Examples are transformation between mechanical energies, and transformation of mechanical energy to work; heat transfer processes and degradation of mechanical energies into heat or heat energy; and production of work to be derived from heat and fuel-energies. The classification of the latter two was also made in Sect. 6.5 of $\left[{ }^{4}\right]$ as spontaneous natural processes and reversible-like processes. In terms of causality undergirding these processes, we can also group processes of the first two kinds into one group while processes of the third kind under a 
distinctive group.

The new materials of this paper are the explication of three points. That processes of the former group can be treated with efficient causation or CVPC. In that context, their treatment is characterized in terms of connection and conversion (i.e., inevitablly conncected) interchangeably. Both are integrated parts of the pre-industrialization "previous habits." This "previous habits" has made it impossible to understand processes of the latter group of unnatural direction correctly (see Sects. 4 and 5 for additional discussion). Whereas the correct understanding of processes of the latter group is exemplified by that of interpreting the Carnot heat engine (and all real heat engines as well, as reversible-like machines) in terms of connection, in accordance with the energy-conservation-principle, andextraction of heat, in accordance with the Carnot-Clausius cycle. That is, the Carnot cycle is to be understood in terms of the Carnot-Clausius cycle, an understanding with the underlying idea of interventionist causation. The very notion of EGP-enabled "extraction," rather than conversion tied to a given outcome, suggests a process of intervention for apossible beneficial-outcome.

\section{Directionality in Thermodynamics}

The rest of the paper, in Sects. 4 and 5, will be a report of outline of a new-synthesis project to be carried out as well as, in Sect. 5, a response to Job and Lankau's critique on equivalence principle.

Joule's theory of heat, equivalence principle, taught that all processes are connected in accordance with the conservation of total energy. The principle itself does not address directionality, nor causality other than the CVPC kind of causality. Directionality or causality of interventionist kind is the purview of the second law.

As the issue of connection vs. conversion addressed in this paper attests, it is not surprising that the directionality issue is complicated. First, Thomson formulated the second law as the principle of degradation of energy. Coppersmith wrote on how Thomson "extended the canvas of the Second Law to ... all of nature,"

... in 1852, all these ideas came together in three bold declarations, stated abruptly at the end of a brief paper entitled 'On a universal tendency in nature to the dissipation of mechanical energy' [xix]:

There is at present in the material world a universal tendency to the dissipation of mechanical energy ... .

Any restoration of mechanical energy, without more than an equivalent of dissipation, is impossible...

Within a finite period of time past, the earth must have been, and within a finite period of time to come the earth must again be, unit for the habitation of man ...

The second declaration is the key one, which Planck concluded to be an invalid truism²1:103-104]. A detailed analysis supporting Planck's conclusion can be found in Sect. 5.10 of $A$ Treatise $\left[{ }^{4}\right]$. The correct assessment is that the principle of energy degradation is subsumed under the entropy growth principle that whereas entropy grows spontaneously and universally, energy degrades spontaneously not universally. This assessment is summarized in Table I. 
Table I_Propositions of the Thomson-Clausius synthesis of the mechanical theory of heat (MTH) and A set of revised propositions

\begin{tabular}{|c|c|c|c|c|}
\hline & & $\begin{array}{l}\text { The Thomson-Clausius } \\
\text { synthesis of MTH }\end{array}$ & & $\begin{array}{l}\text { Thermodynamics, a new } \\
\text { synthesis }\end{array}$ \\
\hline $\begin{array}{l}\text { Ontology (the } \\
\text { ontology of } \\
\text { reality) and its } \\
\text { presupposi- } \\
\text { tions }\end{array}$ & $\begin{array}{l}\text { GS-1.1 } \\
\text { GS- } \\
1.1 \mathrm{a} \\
\text { GS-1.2 }\end{array}$ & $\begin{array}{l}\text { Mechanical conception of heat } \\
\text { providing unity of nature in } \\
\text { terms of } \\
\text { - Universal principle of the } \\
\text { interconversion of natural } \\
\text { powers } \\
\text { Metaphysics of necessity }\end{array}$ & $\begin{array}{l}\text { GS-1.3 } \\
\text { GS-1.3-1 } \\
\text { GS-1.3-2 } \\
\text { GS-1.2-1 } \\
\text { GS-1.2-2 }\end{array}$ & $\begin{array}{l}\text { Mechanical conception of heat in } \\
\text { terms of } \\
\text { - TTS of laws of physics } \\
\text { - Cosmological creation of non- } \\
\text { equilibrium Universe (CCNEU) } \\
\text { Physical necessity } \\
\text { Causal necessity (Interventionist } \\
\text { causation) }\end{array}$ \\
\hline $\begin{array}{l}\text { The first law of } \\
\text { thermo- } \\
\text { dynamics: } \\
\text { Physical }\end{array}$ & $\begin{array}{l}\text { GS-2 } \\
\text { GS-2a }\end{array}$ & $\begin{array}{l}\text { Equivalence principle (MEH): } \\
\text { universal connection } \\
\text { Interconvertibility principle: } \\
\text { universal connection + causation }\end{array}$ & GS-2 & Equivalence principle (MEH) \\
\hline necessity & GS-2.1 & $\begin{array}{l}\text { Principle of energy conversion } \\
(\mathrm{MEH}+\mathrm{GS}-3.1)\end{array}$ & $\begin{array}{l}\text { Amended } \\
\text { GS-2.1 }\end{array}$ & $\begin{array}{l}\text { Principle of energy conservation } \\
\text { (MEH) }\end{array}$ \\
\hline $\begin{array}{l}\text { The second } \\
\text { law of thermo- } \\
\text { dynamics: } \\
\text { causal } \\
\text { necessity }\end{array}$ & $\begin{array}{l}\text { GS-3.1 } \\
\text { GS-3.2 }\end{array}$ & $\begin{array}{l}\text { The energy degradation } \mathbf{X} \\
\text { principle } \\
\text { Universal entropy growth }\end{array}$ & $\begin{array}{l}\text { GS-3.2 } \\
\text { GS-3.3 }\end{array}$ & $\begin{array}{l}\text { Entropy growth selection principle } \\
\text { EGP causal principle }\end{array}$ \\
\hline $\begin{array}{l}\text { How we see/ } \\
\text { interact with } \\
\text { the world in } \\
\text { terms of the } \\
\text { presupposition } \\
\text { of reality and } \\
\text { the two laws }\end{array}$ & $\begin{array}{l}\text { GS-4 } \\
\text { GS-4a } \\
\text { GS-4b }\end{array}$ & $\begin{array}{l}\text { Energy conversion doctrine } \\
\text { Spontaneous energy conversion } \\
\text { Reverse energy conversion: } \\
\text { heat cannot be entirely converted to } \\
\text { work }\end{array}$ & $\begin{array}{l}\text { GS-4.1a(GS- } \\
\text { 4a) } \\
\text { GS-4.1b }\end{array}$ & $\begin{array}{l}\text { Energy conversion/EGP-powered } \\
\text { heat extraction } \\
\text { Spontaneous energy conversion } \\
\text { Reversible-like processes in triadic } \\
\text { framework }\end{array}$ \\
\hline
\end{tabular}

The first point that is summarized in Table $\mathrm{I}$ is that the problematic origin of directionality issue was the interpretation of equivalence principle (MEH), as designated as GS-2 in the table, as the interconvertibility principle, GS-2a. GS-2a had its real origin in the $19^{\text {th }}$ century philosophical doctrine of universal principle of the interconversion of natural powers GS$1.1 \mathrm{a}$, not, in fact, in the empirical equivalence principle. This had strong impact in the eventual formulation of the first law; while the energy degradation principle, GS-3.1, formulated as Thomson's second law, was also partially incorporated into the traditional statement of the first law. The law is referred to in Table I as the principle of energy conversion, GS-2.1. Both the interpretation of equivalence as interconvertibility and the energy degradation truism had strong influence on the traditional understanding of the first law as GS-2.1 by giving it misplaced directionality.

As a result of that, the second law of thermodynamics was deprived of its full meaning of causality, which is the second point summarized in Table I. Although Clausius began "the road to entropy" with implicit interventionist approach, the end result was a universal entropy growth principle, GS-3.2, that has been interpreted as a "selection principle of nature" ([xxi]:4). As a selection principle, it explains the direction of irreversible "processes of natural direction," the kind of processes of interest to physics and chemistry. 
The problem with the Thomson-Clausius synthesis of MTH is that it does not do a good job of explaining the directionality of processes of unnatural direction, more precisely what to do with this directionality, of interest to engineering. Spontaneous "processes of natural direction" just happen, whereas processes of unnatural direction must be made happened. That is, it is necessary to generalize the second law from being a selection principle to a dual principles of entropy growth selection principle, GS-3.2, and entropy growth potential (EGP) causal principle, GS-3.3, [see ${ }^{9}$ for more details]. Here, "causal" means causality of the interventionist causation kind.

By not giving Carnot's theory its full due and making Joule's theory to be its cornerstone, inadequacy of the ThomsonClausius synthesis is in full display in Table I. Since this inadequacy makes understanding of "processes of unnatural direction" impossible and understanding processes of unnatural direction is exactly what energy transition's search for the implication of the NWC discovery of motive power of heat is all about-a new synthesis of Carnot's and Joule's theories is urgently needed.

This paper calls for such a project and argues for the Carnot-Clausius cycle to be a central piece of the project.

\section{Reversibility and the real meaning of equivalence principle}

Demarcation of connection and conversion (in Sect. 2) led to demarcation of the first law and the second law with the former to be a principle of universal connection (the only causality being efficient causality) and the latter a principle of unidirectionality of time and universal causation as shown in Table 1. The identification of unidirectionality of time, i.e., statistical tendency of nature or entropy growth potential (EGP), as causal force for both processes of natural direction and processes of unnatural direction, is a hugely important step: thinking fuel-energy as energy is what has gotten us in trouble in the Anthropocene; we need to rethink fuel-energy in terms of its statistical tendency. There is, however, one more detail about the interventionist nature for applying thermodynamics to processes of unnatural direction: EGP per se does not lead to desired processes of unnatural direction; in other words, EGP and a desired processes of unnatural direction are not dyadically related.

Unlike the cause-effect relation of mechanical energy as cause to produce work, EGP in fuel-energy cannot directly bring about the production of work. For processes of unnatural direction, EGP and work need a third element for the two to be related. Equivalence principle is not "superfluous"! This is where the meaning of equivalence principle is found: the principle of "the production of work is accompanied by the disappearance of an equal-amount-energy of heat means that for EGP to bring about the production of work it needs a heat reservoir so that EGP can enable the extraction of heat from the heat reservoir "delivering" it as work to a work reservoir. EGP, work, and heat reservoir are related in a Borromeanrings triadic interrelationship $[x \times v i i]$. 


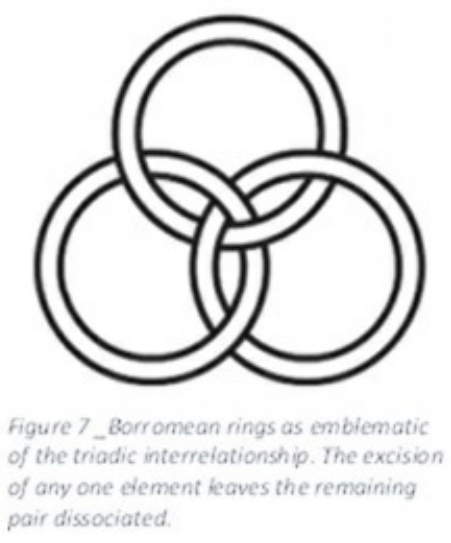

As suggested by the Borromean-rings in Fig. 7, excision of the ring of entropy growth potential leaves the "dyadic" pair of the heat ring and the work ring dissociated. So, we cannot assume a dyadic linkage between heat and work for the cases of heat $\rightarrow$ work change. Excision of heat reservoir also leaves the dyadic pair of EGP and work reservoir dissociated: Without efficacious management of extraction of heat from a heat reservoir, the EGP as the driving force (cause) cannot lead to the production of work (effect) spontaneously.

The second law as a causal principle, GS-3.3, asserts the existence of a new driving force of nature, statistical tendency of nature found in EGP, while the first law, i.e., equivalence of heat and work, prescribes how processes of unnatural direction driven by EGP are brought about in a triadic framework of EGP, heat reservoir, and work.

\section{Conclusion}

There are two distinctive emphases in approach in the Thomson-Clausius synthesis. Thomson based his energy physics on the ideas of conservation of energy and availability of energy. $\left[{ }^{4}\right]$ The approach has dominated the engineering application of thermodynamics, especially the application of exergy analysis to engineering problems. The shortcoming of this approach is that the energy-alone focus is a mistaken focus associated with the erroneous assignment of heat of its "double roles that it cannot fulfill." This has consequences in the aftermath of fuel-energy consumption leading to the Anthropocene.

Clausius based his MTH on the ideas of conservation of energy and "compensation" between "transformations." The latter led to the concept of entropy and entropy principle. His treatment, more precisely the scientific application of his results extended and transformed in the hands of Gibbs, has dominated the scientific application of thermodynamics. This scientific branch-off has been an extraordinarily successful development. Unfortunately, scientific application of thermodynamics lefts out the "heat and work" applications, consequently the entropy principle being accepted as a selection principle solely and the interventionist causation Clausius hinted at with his concept of compensation never becoming explicit in the engineering literature. Significant though scientific application of thermodynamics is, engineering application had been what Carnot had in mind when he founded the science and Clausius too when he began his road to entropy - and engineering understanding of thermodynamics is what we need for getting energy transition right to avoid the worst of the Anthropocene. 
This paper argues for an explicit interventionist-causation treatment of thermodynamic processes of unnatural direction. Thermodynamic processes of unnatural direction were what Carnot sought to address, what Thomson's energy physics failed to explicate because energy physics got stuck in the understanding of mechanical energies of the preindustrialization age, and what Clausius got the right starting point in constructing the six-stage Clausius cycle. It correctly identified that the driving force was the Carnot-proposed "transfer of heat" of $Q_{1}$ from $T_{1}$ to $T_{2}$. The interventionistcausation treatment here revises the cycle to the Carnot-Clausius cycle as shown in Fig. 6b, which shows that the Carnot cycle amounts to the extraction of heat of the amount of area-under-1'2' delivering it as work of the amount of areaenclosed-by-34573/3452'3 (these two areas are equal areas). Thus, a new synthesis based on interventionist-causation treatment of thermodynamic processes of unnatural direction is called for: a new synthesis in accordance with a statistical worldview that upholds the $18^{\text {th }}-19^{\text {th }}$-century discovery of the motive power of heat to be a discovery ofentropy growth potential, not energy. The energy transition implication will be that "entropy pessimism" is an unwarranted notion: inevitability of universal entropy growth does not infer inevitability in heat accumulation nor inevitability in global entropic disorder (see $\left.\left[{ }^{9}\right]\right)$.

The new synthesis will diagnose what troubled the industrialization-based "the Anthropocene" and suggest how we should plan for a "Anthropocene 2.0" without disowning the Anthropocene. More specific consideration of "compensation" as the core concept of the new synthesis and that of the new synthesis' implication to Anthropocene 2.0 will be made in other papers. The energy transition we need today is not a transition away from entropy growth, but a transition from one kind of EGPs, fuel-energies, to another kind of EGPs, solar and wind-for producing economic growth and global entropic order, i.e., sustainable economic growth.

\section{References}

[i] Lovelock J (2019). Novacene: The Coming Age of Hyperintelligence(Allen Lane, an imprint of Penguin Books)

[ii] Bristol T. Reconsidering the Foundations of Thermodynamics from an Engineering Perspective.Preprints 2018, 2018070139 (doi: 10.20944/preprints201807.0139.v1)

[iii] Job G, Lankau T (2003) How harmful is the first law? Ann NY Acad Sci 988:171-181

[iv] Wang L-S (2019). A Treatise of Heat and Energy (Springer, Dec. 7, 2019)

[v] Clausius R (1867) Eighth Memoir in The Mechanical Theory of Heat. p. 290

[vi]Wang L-S (2011). "Causal efficacy and the normative notion of sustainability science,"SSPP 7 (No. 2):30-40

[vii] Sarasvathy S D (2001). "CAUSATION AND EFFECTUATION: Toward a theoretical shift from economic inevitability to entrepreneurial contingency," Academy of Management Review Vol. 26(No. 2): 243-263.

[viii] Ulanowicz R E (2009). "Increasing entropy: Heat death or perpetual harmonies?" Int. J. of Design\&Nature and Ecodynamics (IJDNE) Vol. 4 (2):83-96

[ix] Wang L-S (2021). "Progress in entropy principle,"IJDNE Vol. 16 (No. 4): 359-372

[x] Zwier K R (2014). INTERVENTIONIST CAUSATION IN PHYSICAL SCIENCE_ PhD thesis, University of Pittsburgh 
(November 20, 2014)

[xi] Ulanowicz R E (2021). "Socio-Ecological Networks: A Lens That Focuses Beyond Physics,"Front. Ecol. Evol. Vol. 26 February 2021 | https://doi.org/10.3389/fevo.2021.643122: 1-8.

[xii] DeVreese L (2006). PLURALISM IN THE PHILOSOPHY OF CAUSATION. pp.5-150.

[xiii] Baldwin M, Sobotka A, Clough M P. "Conceptualizing energy," StoryBehindTheScience (Physics Stories section in www.storybehindthescience.org)

[xiv] Nickles T (2020). "Historicist Theories of Scientific Rationality,"Stanford Encyclopedia of Philosophy, p. 3

[xv] Passmore, J., 1967, "Logical Positivism”, The Encyclopedia of Philosophy (Volume 5), P. Edwards (ed.), New York: Macmillan, 52-57

[xvi] Woodward J (2003). Making Things Happen. A Theory of Causal Explanation(Oxford: Oxford University Press) [xvii] Cropper W H (1986). "Rudolf Clausius and the road to entropy,"Am. J. Phys. 54 (12):1068-1074.

[xviii] Clausius, Rudolf. (1850) 1851. "On the Moving Force of Heat, and the Laws regarding the Nature of Heat itself which are deducible therefrom." The London, Edinburgh and Dublin Philosophical Magazine and Journal of Science,4th ser., 2 (8): 1-21

[xix] Coppersmith J (2015). Energy, the Subtle Concept, Revised edition.

[xx] Clausius R $(1854,1867)$. "On a modified form of the second fundamental theorem in the mechanical theory of heat," reprinted as the "Fourth Memoir" in The Mechanical Theory of Heat, translated by T. Archer Hirst (van Voorst, 1867), pp.111-135.

[xxi] Planck M (1969) Treatise on Thermodynamics, 3rd edition. Dover, New York [xxii] Poincaré H (1913). Science and Hypothesis (The Science Press, Lancaster, PA).

[xxiii] Wang, L.S. (2014). "Entropy growth is the manifestation of spontaneity," Journal of Thermodynamics, 2014: 387698. https://doi.org/10.1155/2014/387698

[xxiv] Drake, G W F. "Thermodynamics,"Encyclopaedia Britannica (retrieved September 20, 2019).

[xxv] Thomson W (Lord Kelvin) (1911). Mathematical and Physical Papers of William Thomson 1:511-514. (Cambridge Univ Press)

[xxvi] Ebeling W and Hoffman D (1990). The Berlin school of thermodynamics founded by Helmholtz and Clausius. European Journal of Physics, 12:1-9.

[xxvii] Ulanowicz RE (2019). "The tripartite nature of causalities in ecosystem dynamics,"Current Opinion in Systems Biology 